DOI: http://dx.doi.org/10.22483/2177-5796.2018v20n2p455-470

\title{
Reflexiones sobre el desarrollo del aprendizaje y la complejidad neurocognitiva
}

\author{
Thiago Henrique Barnabé Corrêa \\ Claudia Patricia Moreno Cely \\ Nubia Elena Pineda de Cuadros
}

Resumen: El presente texto pretende acercar y discutir la relación entre experiencia, memoria, emoción y estímulo en el proceso de enseñanza y aprendizaje. Para ello, buscamos apoyo en diversos estudios, los cuales aportan algunos fundamentos sobre los mecanismos cerebrales relacionados al aprendizaje humano. Con eso, a través del diálogo entre los autores citados y sus interlocutores, hacemos algunas consideraciones sobre la complejidad del cerebro humano en lo que denominamos elementos basales del aprendizaje. Aunque este ensayo promueva un tatear inicial por el tema en discusión, buscamos señalar que no se puede asignar la racionalidad del ser humano a un solo factor, pero sí distintos.

Palabras clave: Neurocognición. Plasticidad cerebral. Aprendizaje. Catálisis cognitiva.

\section{Reflexões sobre o desenvolvimento da aprendizagem e a complexidade neurocognitiva}

Resumo: O presente texto pretende abordar e discutir a relação entre experiência, memória, emoção e estímulo no processo de ensino e aprendizagem. Para isso, buscamos apoio em diversos estudos que fornecem alguns fundamentos sobre os mecanismos cerebrais relacionados à aprendizagem humana. Com isso, através do diálogo entre os autores citados e seus interlocutores, fazemos algumas considerações sobre a complexidade do cérebro humano no que denominamos de elementos basais de aprendizagem. Embora este ensaio promova um tatear inicial pelo assunto em discussão, buscamos apontar que não se pode atribuir a racionalidade do ser humano somente a um único fator, mas sim, distintos.

Palavras chave: Neurocognição. Plasticidade cerebral. Aprendizagem. Catálise cognitiva. 
CORRÊA, Thiago Henrique Barnabé; CELY, Claudia Patricia Moreno; CUADROS, Nubia Elena Pineda de. Reflexiones sobre el desarrollo del aprendizaje y la complejidad neurocognitiva.

\section{Concepciones iniciales: la constitución de un cerebro fisio-social}

Aún no se sabe qué factores, internos (genéticos y bioquímicos) o externos, fueron determinantes para conducir a nuestros ancestros a desarrollar un cerebro mayor en complejidad sináptica, así como, en complejidad interacional (social). Aunque el cerebro sea una estructura común a los mamíferos, una cosa nos intriga cuando retratamos al ser humano: la singularidad de su actividad consciente, siendo un desafío para aquellos que buscan comprenderlo, sobre todo, cuando nos referimos a lo que pasa en el nivel de la conciencia.

Luria (1979) nos ayuda a pensar sobre la actividad consciente del hombre puntuando tres trazos fundamentales de éstas. El primero de estos consiste en que la actividad del hombre no está obligatoriamente vinculada a motivos biológicos. El segundo trazo característico de la actividad consciente del hombre consiste en que no es forzosamente determinada por impresiones evidentes, recibidas del medio o por vestigios de la experiencia individual inmediata. El tercer trazo es que la gran mayoría de los conocimientos y habilidades del hombre se forma por medio de asimilación de la experiencia de toda la humanidad, acumulada en el proceso de la historia social y transmisible en el proceso de aprendizaje. Apoyado en estos, este ensayo teorico reconoce al sujeto como el producto total de un cerebro complejo en su fisiología, pero también de un cerebro "social" que se constituye en la relación con el Otro.

Bajo la perspectiva interaccionista-cognitivista, el desarrollo cognitivo ocurre con el fundamento del modelo social a través del aprendizaje en constante interacción sujeto-medio. A través de la interacción con el medio, e incluimos la interacción con el Otro, el sujeto presenta condiciones para una agitación cognitiva interna hacia el estado de equilibrio orgánico [homeostasis]. A medida que el sujeto sufre influencia interna y externa (medio y el Otro) el desequilibrio es restablecido, lo que exige reorganización por dos mecanismos: asimilación y acomodación de la nueva experiencia/información adquirida. El nuevo contenido es de fundamental importancia en nuevas situaciones a él presentadas, así servirá de base para abstracciones más complejas. Por tanto, podemos comprender la interferencia del medio como facilitador de las construcciones cognitivas de la inteligencia, donde se entiende la cognición como el proceso de conocer.

Frente a lo expuesto, no estamos ignorando la programación genética del sujeto, pero enfatizamos que el carácter mutable y flexible de las interconexiones neurales frente al estímulo 
del medio se atribuye a la modulación de la condición del medio y la necesidad de cambio del organismo (supervivencia de éste) ya que entendemos la necesidad como una propiedad real del medio (interna y externa al sujeto). Esto nos lleva a pensar la inteligencia humana - la racionalidad - un discontinuo cognitivo multidireccional modulado por distintas facultades mentales y por la relación fisiológica, emocional, ambiental e interactiva.

Sobre la rica capacidad dialógica de interacción humana es evidente que reconocemos el valor del lenguaje y su importancia decisiva para la posterior reorganización de la actividad consciente del ser humano, considerando ésta, en asociación con las relaciones sociales estabelecidas por el hombre, como el trabajo, un factor fundamental para la formación de la conciencia, de la promoción de la interactividad y, por consiguiente, del aprendizaje. Sin embargo, hacemos por elección en este texto no explotarla.

En esta óptica, el presente texto pretende acercar y discutir la relación entre experiencia, memoria, emoción y estímulo en el proceso de enseñanza y aprendizaje. Para ello, buscamos apoyo en los estudios de Siqueira y Gurgel-Giannetti (2011), Guerra (2011), Izquierdo (2011) y Oliveira (2015), los cuales aportan algunos fundamentos sobre los mecanismos cerebrales relacionados al aprendizaje humano. Con eso, a través del diálogo entre los autores citados y sus interlocutores, hacemos algunas consideraciones sobre la complejidad del cerebro humano en lo que denominamos elementos basales del aprendizaje.

\section{Nuances de aproximaciones entre Aprendizaje y Neurociencia}

El tema del aprendizaje también ha sido objeto de investigación por parte de la psicologia, donde el cerebro sigue siendo un misterio para la ciência a pesar de varios hallazgos. Es complejo determinar como se crea la sinapsis que sucede en la mente de quien aprende generando sensaciones, percepciones, pensamientos y emociones expresadas en los contextos socioculturales a partir del proceso enseñanza y aprendizaje. Según Ortiz Ocaña (2014, p. 40), “el cerebro es dinâmico, creativo y activo, aprende por sí sólo y en la interacción con el medio, su naturaleza es bioneuropsicosocial (genética y cultural)"; eso significa decir, que el resultado del aprendizaje es la mente del ser humano.

De igual forma, las investigaciones sobre Neurociencias han traído contribuciones significativas para la comprensión de la mente humana, principalmente, en lo que se refiere la 
CORRÊA, Thiago Henrique Barnabé; CELY, Claudia Patricia Moreno; CUADROS, Nubia Elena Pineda de. Reflexiones sobre el desarrollo del aprendizaje y la complejidad neurocognitiva.

adquisición del conocimiento. La comprensión del cerebro como órgano responsable para el aprendizaje no es novedad, sin embargo, investigaciones neurocientíficas han acrecentado para la comprensión de cómo ocurren los procesos neurales que intervienen en el aprendizaje (CRESPI; FINIMUNDI, 2016). En este escenario emergen elementos internos (endógenos) y externos (exógenos) al proceso de aprendizaje como cuestiones acerca de las estrategias en el aula y las formas de evaluación adoptadas.

Aunque la Neurociencia y Educación se comuniquen, Oliveira (2011, p. 21) indica la necesidad de diferenciarlas, donde Neurociencia se constituye como "la ciencia del cerebro" y la Educación como "ciencia de la enseñanza y del aprendizaje”. Para el autor, ambas tienen una relación, entendiendo esta relación como un vínculo de proximidad entre el cerebro humano y el proceso cognitivo, tomando un significado más relevante, haciéndolos dimensiones altamente articuladas. En relación con lo anterior, Simões (2016) nos llama la atención, enmarcando que las dos áreas (Neurociencia y Educación) tienen formas de acción y finalidades distintas, por eso, la autora establece que los productos de la neurociencia no se aplican directa e inmediatamente a la educación, necesitando ser "readaptados", donde preferimos decir reinterpretados, para que puedan contribuir efectivamente al campo de la educación. Como trae Guerra $(2011$, p. 4):

La neurociencia puede informar a la educación, pero no explica o proporciona prescripciones, recetas o procesos que van a garantir resultados. Las teorías psicológicas basadas en los mecanismos cerebrales involucrados en el aprendizaje pueden inspirar objetivos y estrategias educativas. El trabajo del educador puede ser más significativo y eficiente si conoce el funcionamiento cerebral, lo que le posibilita el desarrollo de estrategias pedagógicas más adecuadas.

Es evidente que las teorías neurocientíficas pueden ser consideradas una valiosa aliada para las teorías educativas que dejan de estar exclusivamente en el plano abstracto, dando materialidad a los elementos subjetivos de la mente. Es importante decir que los trabajos sobre neurociencias no están restringidos a la anatomía cerebral, donde reducirlos sería lo mismo que ignorar la esfera antropológica (social, cultural e histórica) también consolidada en el campo de la psicología, la educación, la lingüística, entre otras.

Además de asumir la dimensión biológica-fisiológica del cerebro humano, considerando la química detrás del funcionamiento cerebral, este trabajo reconoce, sobre todo, sus marcas sociales (aquellas construidas en la relación con el Otro) y por qué no decir culturales, o sea, su dualidad. En vista de eso, es evidente que tales componentes son indisociables cuando se tiene 
CORRÊA, Thiago Henrique Barnabé; CELY, Claudia Patricia Moreno; CUADROS, Nubia Elena Pineda de. Reflexiones sobre el desarrollo del aprendizaje y la complejidad neurocognitiva.

como objetivo comprender las relaciones de enseñanza como un tejido complejo sin la disyunción de lo biológico-social. Esto ofrece aporte a las reflexiones aquí propuestas, además de atribuir alta complejidad a los estudios sobre los procesos cognitivos en una perspectiva inter y transdisciplinaria. En este, es importante destacar que no se puede simplemente considerar la (co)existencia de la esfera social, pero también debemos pensar en su engendramiento con la maquinaria biológica, principalmente si asumimos que toda experiencia de aprendizaje se inicia con una experiencia afectiva, ya que el afecto se relaciona directamente con las emociones que, a su vez, poseen mayoritariamente origen bioquímico (LeDOUX, 2001).

Dentro del proceso de enseñanza y aprendizaje es importante para el educador conocer el funcionamiento del cérebro ya que desde los estudios científicos se demuestra que el cerebro regula la conducta humana; donde lo endógeno también se manifiesta en lo exógeno, esto en razón de lo que experimentamos en la vida, muchas de las situaciones son dificiles de controlar y regular desde nuestro cerebro, el cual ha venido siendo moldeado o configurado también por elementos externos al cuerpo. La mente humana se concibe como una configuración de configuraciones afectivas, cognitivas e instrumentales. Como señala Ortiz Ocaña (2014, p. 41), "lo afectivo media lo cognitivo, porque lo precede, lo conduce y guía su desarrollo, lo afectivo integrado a lo cognitivo se lleva a la práctica mediante el instrumental"; lo mismo es decir que entre el cérebro y la mente humana se genera una interacción dialógica, dialéctica y configuradora.

Desde los tiempos antiguos, filósofos y pensadores como Platón (428-347 a.C), Descartes (1596-1650) y Kant (1724-1804) ya suponían una separación entre emoción y razón, atribuyendo aún superioridad de valor a esta última. Kant ya decía sobre la imposibilidad del encuentro entre razón y felicidad, afirmando que si Dios hubiera creado al hombre para ser feliz, no lo habría dotado de razón, considerando la pasión como uma enfermedad del alma (de la mente). Esta desvalorización del cerebro fue descrita, por ejemplo, por los egipcios y los asirios que atribuían al hígado el centro del pensamiento, así como Aristóteles que creía que el cerebro sólo servía para enfriar la sangre.

Por influencia de estos pensamientos dicotómicos, la psicología, por mucho tiempo, también estudió los procesos cognitivos y afectivos de manera separada, siendo el suizo Jean Piaget (1896-1980) uno de los primeros nombres a cuestionar la separación entre cognición y afectividad. A través de su obra Les relations entre l'inteligence et l'affectivité dans le 
développement de l'efant, Piaget (2006) afirma que afectividad y cognición son diferentes en naturaleza, pero inseparables en todas las acciones humanas. Toda acción y pensamiento comprenden un aspecto cognitivo, que son las estructuras mentales, y un aspecto afectivo que sirve como una energía. En general, la afectividad sería, para Piaget, funcional para la inteligencia: ella es la fuente de energía por la cual la cognición funciona. Así ¿es cierte afirmar que antes de la razón hay una emoción?

El bielorruso Lev Vygotsky (1896-1934) fue otro que estudió las relaciones entre afecto y cognición, afirmando que las emociones forman parte activa en el funcionamiento mental general. A través del estudio del desarrollo del lenguaje (sistema simbólico utilizado por todos los humanos), Vygotsky estudió los orígenes del psiquismo humano a través de un enfoque unificador entre cognición y afectividad. Uniendo también la razón y la emoción, el francés Henri Wallon (1879-1962) buscó comprender las emociones a través de sus funciones, dándoles un papel fundamental en la evolución de la conciencia de sí. Para él, la evolución de la afectividad depende de las construcciones realizadas en el plano de la inteligencia; La evolución intelectual depende de las construcciones afectivas. Sin embargo, para Wallon, hay fases en que una es más predominante que la otra.

En la actualidad, la visión más discutida sobre la relación entre las emociones y la cognición es la del portugués Antonio Damasio que la diferencia de los demás pensadores sobre este tema y muestra algunas relaciones existentes entre la biología del cuerpo humano (físiología) y el pensamiento. Basado en las ideas de Piaget y Vygotsky, Damasio afirma que las emociones y la razón no son elementos completamente disociados, haciendo la frase del francés Blaise Pascal (1623-1662) un dilema que todavía nos inquieta: "El corazón tiene razones que la propia razón desconoce".

Hasta hoy, el sentido común trae la razón siendo contraria a la emoción, sin embargo, Damasio (2006) muestra en sus trabajos que personas que poseen alguna deficiencia en la región del cerebro responsable por las emociones presentan dificultades de aprendizaje. Esto nos lleva a decir que las emociones no son actos racionales, sino que son las causantes (in)directas de la cognición, siendo fundamentales en el proceso de aprendizaje, ya que las emociones generan sentimientos, que a su vez generan actos racionales y desencadenan el proceso cognitivo.

Según la neurobióloga Candace Pert, toda emoción que sentimos circula por nuestros cuerpos como sustancias químicas llamadas "neuropéptidos" - cadenas cortas de aminoácidos o 
proteínas que se comunican con todas las células de nuestro cuerpo. En sus investigaciones Pert sugiere que esas moléculas de emoción desempeñan un importante papel en orientar lo que vivimos como percepción y elección consciente. Así nuestras emociones deciden a lo que vale la pena prestar atención. La decisión sobre lo que se convierte en un pensamiento que surge en la conciencia y lo que permanece como un patrón de pensamiento indigesto y enterrado en un nivel más profundo del cuerpo es medido por los receptores de nuestra red de informaciones corporal y bioquímica.

De hecho, no desconsideramos la importancia biológica de las conexiones dendríticas y sinapsis constituyendo el contacto funcional entre neuronas y proporcionando el aprendizaje. Sin embargo, este importante mecanismo neuroanatomofisiológico es complementario a los sistemas funcionales complejos y diferenciados y a la influencia de variados estímulos del mundo exterior, en una relación activa sociocultural, modificable históricamente (BASTOS; ALVES, 2013, p. 43).

Ante estas perspectivas, ¿sería posible disociar, separar, la emoción de la razón? Aunque el hombre sea considerado racional, ¿cuál sería nuestro entendimento de la emoción sobre la racionalidad? ¿Y la capacidad de juzgar, medir y calcular o de producir saber/conocimiento? Determinar (si eso es posible) la zona limítrofe entre ambas parece ser la primera caja negra de la neurociencia y la clave para entender nuestra singularidad evolutiva.

\section{Discutiendo las bases del aprendizaje}

¿De qué modo la Neurociencia puede ofrecer a los docentes saberes científicos que se centran en los procesos por los que el cerebro aprende? ¿Cómo se conservan los conocimientos en la memoria? Sabemos que estas preguntas son audaces, pero nos desafiamos en un diálogo inicial. Para eso, necesitamos discutir a qué se refiere el término memoria y cuál es su importancia para el proceso de aprendizaje, considerando que sin la memoria no podríamos construir nuevos conocimientos ni tener acceso a lo aprendido.

De esta manera, asumiremos que las memorias provienen de experiencias y que la sinergia de éstas es el principal generador del aprendizaje. A través del sistema de archivo de las experiencias el ser humano construye su conocimiento y lo hace más complejo. Según D’Aquili (1973), la memoria, o matriz cognitiva, tiende a ser permanente, y gracias a ella el sujeto posee el referencial para construir nuevas percepciones partiendo de los recortes por él vivenciados. 
CORRÊA, Thiago Henrique Barnabé; CELY, Claudia Patricia Moreno; CUADROS, Nubia Elena Pineda de. Reflexiones sobre el desarrollo del aprendizaje y la complejidad neurocognitiva.

Conforme defiende Simões (2016) e Izquierdo (2011), es el conjunto de nuestras memorias que nos individualiza, haciendo que cada sujeto sea único, singular.

La habilidade de aprender y de recordar información acerca del mundo a nuestro alrededor y de nuestras experiencias que en él ocurren es una habilidad cognitiva fundamental que poseemos. Increíblemente, almacenamos millones de informaciones, algunas veces con facilidad, otras veces con mucho esfuerzo (GAZZANIGA, 2006, p. $320)$.

También vale decir que el aprendizaje está acoplada a las emociones, y que almacenamos, principalmente, lo que nos marca, nos emociona. A lo largo del tiempo, lo que no nos interesa va siendo olvidado o incluso "adormicido" mientras que vamos incorporando nuevas informaciones y almacenando otras que nos es significativa. Con eso, tenemos la capacidad de dar espacio para la superposición del nuevo conocimiento, de modo que incorporamos nueva información y almacenamos otras que nos es significativa. En otras palabras, aprendemos y aprehendemos lo que nos tiene sentido en un determinado contexto en el que vivimos, que tenga vínculos con lo que ya es conocido, que atienda a expectativas, o sea estimulante y agradable.

Para Guerra (2011), aprendemos lo que es útil para nuestra supervivencia y/o que nos proporciona placer. Pero también no podemos olvidar que aprendemos cuándo nos interesa, cuándo sentimos necesidad. Todos esas ideas estan lejos de la escuela actual donde el aprendizaje difiere de la visualización de utilidad en los contenidos escolares que, exclusivamente, sierven para "merecer" nota o pasar los exámenes. Es por eso que para la referida autora, la evolución nos ha garantizado un cerebro capaz de aprender a fin de proporcionar nuestro bienestar y supervivencia, y no para tener éxito en la escuela.

El placer y el aprendizaje forman una pareja complementaria en la escuela, ya que sabemos que el neurotransmisor dopamina tiene íntima relacion con ellos. Producida cuando experimentamos uma experiencia placentera o una recompensa agradable, ese neurotransmisor también afecta el aprendizaje que permite que el cerebro capte y retenga más información. Decimos eso por que hemos asumido que el primer papel de la escuela es despertar la curiosidade y estimular en los estudiantes el placer en buscar conocimiento, en aprender, y, además, preguntar y duvidar, cosa que poco se ha promovido.

El estímulo es otro elemento importante y un eslabón a ser ponderado entre la memoria y el aprendizaje. Esto nos hace pensar en las estrategias de enseñanza adoptadas en el aula, que a menudo son carentes o incluso ausentes de estímulos suficientes para desencadenar un 
aprendizaje efectivo y/o relevante. Son estos (los estímulos) que permiten lo que la Neurociencia llama plasticidad cerebral o neuroplasticidad: establecimiento de nuevas conexiones y la adaptación a nuevas situaciones, a partir de las necesidades creadas o existentes en el medio (OLIVEIRA, 2015). La plasticidad cerebral es un mecanismo muy importante para el entendimiento de los conceptos Piagetianos de asimilación y acomodación de nuevas informaciones, principalmente cuando consideramos la asimilación una interpretación selectiva de esas y la acomodación el proceso de reorganización mental.

Izquierdo (2011) denomina plasticidad cerebral el conjunto de procesos fisiológicos, a nivel celular y molecular, que explica la capacidad de las células nerviosas de cambiar sus respuestas a determinados estímulos en función de la experiencia. Para Guerra (2011, p. 6) la "neuroplasticidad es la propiedad de 'hacer y deshacer' conexiones entre neuronas. Que permite la reorganización de la estructura del sistema nervioso y del cerebro y constituye la base biológica del aprendizaje y del olvido".

Cuando nos referimos al estímulo en el proceso de aprendizaje vale decir que se hace importante que éste sea de naturaleza diversa, ya que el uso recurrente del mismo estímulo acaba por condicionar y adaptar el cerebro a la misma respuesta. Es por este motivo que estímulos diferentes son capaces de "ejercitar" las neuronas y ampliar la capacidad sináptica en el proceso cognitivo. Los estímulos variados y las prácticas (pedagógicas) que privilegian la reflexión y la argumentación son capaces de ampliar la capacidad que el cerebro tiene de adaptarse a una nueva condición/situación. Todo esto es posible gracias a la compleja y eficiente plasticidad cerebral. En síntesis, traemos en la figura 1 un esquema sencillo de los elementos descritos y su articulación.

Figura 1 - Esquema simplificado de la relación experiencia-aprendizaje

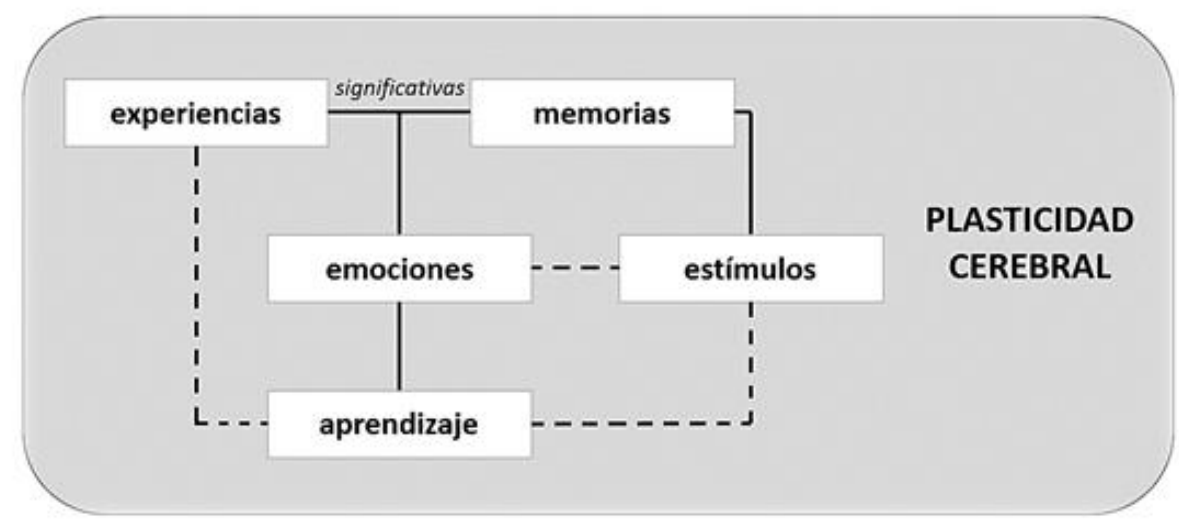

Fuente: Del autor. 
Siqueira y Gurgel-Giannetti (2011) afirman que la plasticidad cerebral en los niños es de extrema importancia para el aprendizaje, ya que es en la infancia que hay mayor maleabilidad del cerebro humano, estando él con mayor "predisposición" para aprender, favoreciendo la adquisición de nuevos conocimientos y funciones. Como el cerebro es capaz de reorganizarse en torno de las conexiones neurales relevantes para su adaptación al paso en que se encuentra el medio, es necesario reconocer que la motivación/estímulo ejerce gran impacto en el aprendizaje, siendo que "cuanto más interesante e importante es la información, más fácil su retención y rescate cuando sea necessário" (p. 79).

Con eso, necesitamos revisar la creencia de que 'aquello que no se aprende cuando niño tampoco se aprenderá cuando adulto', pues, incluso después de la infancia las redes neurales todavía estarán dispuestas a la neuroplasticidad, aunque el diagrama básico de los circuitos formados por las células nerviosas ya estea constituido. El concepto de plasticidad cerebral permite el entendimiento de que el cerebro continúa desarrollándose, cambiando y renovándose durante toda la vida del ser humano. Esto nos permite comprender que las personas poseen potencialidades para aprender desde su nacimiento hasta su muerte, siendo necesario para tanto disponer las condiciones para que eso ocurra. Lo expuesto va al encuentro de Freire (2008, p. 47), el cual afirma que "enseñar no es transferir conocimiento, sino crear las posibilidades/condiciones para su propia producción o su construcción”.

En suma, es esencial al profesor comprender la existencia de la plasticidad cerebral, que es la capacidad del sistema nervioso de adaptarse a nuevos estímulos e influencias, permitiendo la flexibilización necesaria para aprender. Es a partir de la conciencia de este mecanismo que el profesor va a entender cómo las estructuras cerebrales se reorganizan a cada nuevo aprendizaje, resultando en cambios. Lent (2010) alerta que hay que considerar que estos cambios (estructurales y funcionales del sistema nervioso) producen efectos en el comportamiento y en el desarrollo psicológico del individuo, lo que nos lleva a decir que estos cambios también desencadenan una plasticidad conductual (social). A partir de estas conjeturas ¿podríamos decir entonces que el resultado del aprendizaje es la mente del ser humano? ¿O el inverso?

Hablar sobre el aprendizaje es un desafío para los educadores pues aunque la interacción social sea un elemento importante para este proceso, en última instancia, conforme pontualiza Cosenza y Guerra (2011), el aprendizaje es un fenómeno individual y privado que obedece a las 
CORRÊA, Thiago Henrique Barnabé; CELY, Claudia Patricia Moreno; CUADROS, Nubia Elena Pineda de. Reflexiones sobre el desarrollo del aprendizaje y la complejidad neurocognitiva.

circunstancias históricas de cada uno, lo que demuestra también el carácter inconciente de los procesos cognitivos.

\section{La catálisis cognitiva}

Como ya citado sobre la agitación cognitiva y el movimiento de desiquilibrio para alcanzar el nuevo equilíbrio de las ideas, se asume que las transformaciones cerebrales en el proceso de aprendizaje obedecen a la segunda Ley de la Termodinámica, donde existe una tendencia del sistema a aumentar el grado de desorden (Entropia, $\boldsymbol{S}$ ), o sea, de tender al caos. Aunque este concepto se emplea en la Física y la Química, buscamos apoyo en este a fin de representar las ideas en lo que llamamos de catálisis cognitiva.

La entropía es una grandeza termodinámica relacionada con el grado de desorden del sistema, así, a partir de una analogía, defendemos que el proceso de aprendizaje requiere desorden y abandono del estado de inercia mental, o sea, el proceso de aprendizaje necesita de confusión: de confrontación de ideias y puntos de vista. Para esta teoría, cuanto mayor sea el $S$, mayor el desorden, lo que puede ser traducido al aprendizaje, de modo que cuanto mayor la confusión y el choque de ideas, mayor es la posibilidad de aprender. Esto nos da libertad para considerar que el segundo papel de la escuela es ayudar los estudientes a superar el estado de inercia y promover el desorden de pensamiento, es enseñar a los estudiantes a hacer preguntas para respuestas que no se han dado, y no lo inverso, como observamos en la cultura escolar: dar respuestas a preguntas que no fueron realizadas.

Al encuentro de estas palabras, defendemos que ser profesor va mucho más allá de enseñar un contenido: es ser capaz de despertar y catalizar para lo nuevo; es desnaturalizar ideas listas; es promover la ampliación del universo cultural y una mirada reflexiva, consciente y (auto)crítica de la realidad (CORRÊA, 2015).

El docente como ese ser que conoce y promueve en el estudiante la crisis cognitiva (de ideas) y la escuela como espacio en donde se recrea la interacción mental, social y cultural, que es la vida real de lo cotidiano. Realidad que varia en docentes y estudiantes, por su conocimiento y práxis. Como afirma Marrero (2012, p. 4-5), "la información ya no se encuentra encapsulada en módulos particulares, sino que, sin perder la organización modular, la fluidez cognitiva alcanzada a partir de la aparición del lenguaje, permite una integración de diferentes tipos de información, 
CORRÊA, Thiago Henrique Barnabé; CELY, Claudia Patricia Moreno; CUADROS, Nubia Elena Pineda de. Reflexiones sobre el desarrollo del aprendizaje y la complejidad neurocognitiva.

es decir, el mundo social con el mundo no social. De esta forma, el lenguaje y la fluidez cognitiva permiten percepciones y cogniciones más complejas sobre el mundo y sobre nosotros mismos", (WONG, 2005) citado en Marrero (2012). Por eso, es a través del lenguaje (escrita, oral y gestual) que transmitimos conocimientos o somos capaces de despertar una emoción.

Aunque este ensayo promueva un tatear inicial por el tema en discusión, buscamos señalar que no se puede asignar la racionalidad del ser humano a un solo factor, pero sí distintos. Creemos, además, que el desarrollo del conocimiento es un proceso espontáneo ligado al proceso global de la embriogénesis. La embriogénesis se refiere al desarrollo del cuerpo, pero también al desarrollo del sistema nervioso y al desarrollo de las funciones mentales (PIAGET, 1972). De esa manera entendemos que la embriogénesis sólo termina con la muerte del sujeto.

El aprendizaje sigue el binomio aprender-aprehender, pues se da de forma opuesta a la embriogénesis. El aprendizaje es aquí entendida como un proceso no espontáneo, cuyo desarrollo es provocado por situaciones y catalizado por experiencias, vivencias y, también, por el profesor. Esto nos lleva a decir que el aprendizaje es un conjunto de la maduración cerebral, de experiencia física y social y del principio del equilibrio, donde la experiencia da origen a nuevas estructuras mentales que amplían el potencial de originar otras "nuevas estructuras mentales". Esto nos lleva a decir que la palabra aprender, en esta perspectiva, no puede ser entendida como sinónimo de apreender, ya que presupone la idea de tomar para sí lo enseñado, convirtiendo lo prendido en aprehendido, o sea, en tuyo. Por esta razón, la enseñanza y el aprendizaje no es una condición sine qua non.

Por último, el aprendizaje puede entenderse con un estado diferente e irreversible del que el sujeto partió, estado que exige la superación de una barrera "energética" que al ser superada desencadena el proceso de aprendizaje. Delante de lo expuesto resumimos nuestras ideas en la célebre e icónica frase de Albert Einstein: la mente que se abre a una nueva idea jamás vuelve a su tamaño original.

Frente a lo expuesto, encerramos este trabajo citando al educador brasileño Paulo Freire en sus reflexiones:

Conocer, en la dimensión humana, que aquí nos interesa, cualquiera que sea el nivel en que se dé, no es el acto a través del cual un sujeto, transformando en objeto, recibe, dócil y pasivamente, los contenidos que otro le da o impone. El conocimiento, por el contrario, exige una presencia curiosa del sujeto frente al mundo. Requiere su acción transformadora sobre la realidad. Demanda una búsqueda constante. Implica en invención y en reinvención [...] Por eso mismo, en el proceso de aprendizaje, sólo 
aprende verdaderamente al que se apropia de lo aprendido, transformándolo en aprehendido, con lo que puede, por ello mismo, reinventarlo; el que es capaz de aplicar lo aprendido-aprehendido a situaciones existenciales concretas ... Por el contrario, aquel que es "llenado" por otro de contenidos cuya inteligencia no percibe; de contenidos que contradicen la forma propia de estar en su mundo, sin que sea desafiado, no aprende (FREIRE, 2015, p. 28).

"La educación es una forma de intervención en el mundo" - Freire (2008, p. 93), de transformación de realidades vividas en los distintos contextos escolares, en los cuales se debe manifestar la emancipación del sujeto en donde todo conocimiento, saber y experiencia redundan en la formación del otro como ser sociable y constructor de paz. Es así, que la mente y el cerebro humano, la razón, la ética, la política y demás ciencias deben apuntar a la evolución del que aprende a través de dispositivos pedagógicos, operacionalidad cerebral, sinapsis y muchos otros procesos mentales con el fin de hacer aportes a los cambios que implica la tecnología, la ciencia y la educación.

\section{Consideraciones finales}

Como se puede observar, la complejidad de las funciones cerebrales demuestra los desafíos de interpretar los mecanismos del aprendizaje, revelando un cerebro transitorio: racional y emocional. Aunque trae sólo algunos elementos a considerar, este trabajo no excluye la importancia de otras facultades mentales como la abstracción, abriendo espacio para otras discusiones.

Consideramos durante toda la construcción de ese ensayo la memoria "biológica" del ser humano, pero apuntamos para la necesidad de investigaciones futuras discutir sobre la memoria virtual presente en el siglo XXI. Hablamos siempre de una nueva tecnología pero olvidamos en nuestros trabajos de hablar de un nuevo sujeto: el Homo sapiens technologicus.

¿Cómo hablar de conocimiento cuándo éste se ha convertido en una dimensión abitótica (nube computacional) - externa al medio cerebral - y independente del Otro? ¿Cómo queda la afectividad cuando pensamos en educación a distancia o virtual?

Es evidente que el desarrollo cognitivo de la nueva generación de personas (generación Z: nativas digitales) pasa por caminos desconocidos y su contacto con las tecnologias y las formas de tratar la información - el conocimiento - afectan e influencian la dinámica sináptica y activan distintas áreas cerebrales, haciendo con que la manera como aprendemos hoy sea diferente y 
CORRÊA, Thiago Henrique Barnabé; CELY, Claudia Patricia Moreno; CUADROS, Nubia Elena Pineda de. Reflexiones sobre el desarrollo del aprendizaje y la complejidad neurocognitiva.

porque no decír más compleja. ¿Como hablar entonces de la influencia del Otro en el proceso de aprendizaje cuando su presencia es indirecta o mismo ausente (virtual)?

¿Estamos frente a las limitaciones de las teorías del aprendizaje? ¿Cómo aprendemos a aprender? Todas esas preguntas son necesarias cuándo consideramos la dinámica de mundo y la volatilidad de las ideas, principalmente cuándo vivimos una nueva ruptura (paradigma) sociocultural que hace una vez más el pulgar opositor un símbolo de la evolución humana, utilizándolo para arrastrar nuestras pantallas táctiles (touchscreen). Por eso, no hay duda de que inventamos la tecnología y ella nos reinventó.

Después de estas provocaciones no es exagerado decir que los nuevos tempos traem más preguntas y la necesidad de nuevas respuestas para como aprendemos, apuntando no más para una revolución neurocognitiva sino una revolución neuroconectiva. En la verdade tenemos mucho que avanzar, ya que comprendemos poco de nuestros procesos mentales y ya hablamos de inteligencia artificial. Por eso, defendemos en ese texto la urgente búsqueda en romper con la fragmentación, la mutilación y la reducción que la mirada unidimensional del paradigma simplificador lanza sobre los procesos de enseñanza y aprendizaje.

\section{Referências}

BASTOS, L. S.; ALVES, M. P. As influências de Vygotsky e Luria à neurociência contemporânea e à compreensão do processo de aprendizagem. Revista Práxis, Rio de Janeiro, v. 5, n. 10, p. 41-53, dez. 2013.

CRESPI, L.; FINMUNDI, M. Neurociência na prática pedagógica: aproximando fundamentos neurobiológicos da aprendizagem à formação inicial do docente da educação infantil e anos iniciais do ensino fundamental. In: CONGRESSO INTERNACIONAL DE EDUCAÇÃO “Cotidiano escolar: (in)quietudes e fronteiras em conhecimentos e práticas educacionais", 1., 2016, Sorocaba. Anais... Sorocaba, 2016. p. 309-315. Disponível em: <http://uniso.br/publicacoes/anais-internacional-deeducacao/anais-congresso-educacao-05-10.pdf>. Acesso em: 9 dez. 2017.

CORRÊA, T. H. B. Sonhar e ensinar: tarefas de um professor. In: CORRÊA, T. H. B. (Org.). A educação como arte de sonhar. Curitiba: CRV, 2015.

COSENZA, R. M.; GUERRA, L. B. Neurociência e educação. Porto Alegre: Artmed, 2011.

DAMASIO, A. El error de Descartes. Madrid: Crítica, 2006.

D'AQUILI, Eugene G. The biopsychological determinants of culture. In: ADDISON-WESLEY Module in anthropology. 3 ed. Reading, Massachusetts: Addison-Wesley Publishing Company, n. 13, p. 1-29, 1973.

FREIRE, P. Extensão ou comunicação? 17. ed. São Paulo: Paz e Terra, 2015. 
CORRÊA, Thiago Henrique Barnabé; CELY, Claudia Patricia Moreno; CUADROS, Nubia Elena Pineda de. Reflexiones sobre el desarrollo del aprendizaje y la complejidad neurocognitiva.

FREIRE, P. Pedagogia da autonomia: saberes necessários à prática educativa. São Paulo: Paz e Terra, 2008.

GAZZANIGA, M. (Org.). Neurociência cognitiva: a biologia da mente. Porto Alegre: Artmed, 2006.

GUERRA, L. B. O diálogo entre a neurociência e a educação: da euforia aos desafios e possibilidades. Revista Interlocução, Belo Horizonte, v. 4, n. 4, p. 3-12, 2011.

IZQUIERDO, I. Memória. Porto Alegre: Artmed, 2011.

LeDOUX, J. E. O cérebro emocional: os misteriosos alicerces da vida emocional. Rio de Janeiro: Objetiva, 2001.

LENT, R. Cem bilhões de neurônios: conceitos fundamentais de neurociência. São Paulo: Atheneu, 2010.

LURIA, A. R. A atividade consciente do homem e suas raízes histórico-sociais. In: LURIA, A. R. Curso de psicologia geral. Rio de Janeiro: Civilização Brasileira, 1979. p. 71-84. v.1.

MARRERO, A. L. A. La teoría cognitiva y su impacto en el proceso de enseñanza-aprendizaje del ser humano. 2012. Disponivel em: <https://es.slideshare.net/Luis1280/la-teora-cognitiva-y-su-impactoen-el-proceso-de-enseanza-aprendizaje-del-ser-humano>. Acesso em: 11 set. 2017.

OLIVEIRA, G. G. A pedagogia da neurociência: ensinando o cérebro e a mente. Curitiba: Appris, 2015.

OLIVEIRA, G. G. Neurociências e os processos educativos: um saber necessário na formação de professores. 2011. Dissertação (Mestrado em Educação) - Programa de Mestrado em Educação, Universidade de Uberaba - UNIUBE, Uberaba, 2011.

ORTIZ OCAÑA, A. Cerebro, currículo y mente humana: psicología configurante y pedagogía configurante. Barranquilla, Colombia: Ediciones Litoral, 2014. (Colección: Neuropsicología, Didáctica y Pedagogía del Cerebro 1).

PIAGET, J. Development and learning. In: LAVATELLY, C. S.; STENDLER, F. Reading in child behavior and development. New York: Hartcourt Brace Janovich, 1972.

PIAGET, J. Les relations entre l'inteligence et l'affectivité dans le développement de l'efant. Genève, Suisse: Fondation Jean Piaget, 2006. Disponível em:

<http://www.fondationjeanpiaget.ch/fjp/site/textes/VE/JP_54_cours_affect.pdf>. Acesso em: 2 dez. 2017.

SIMÕES, E. M. S. A presença dos conhecimentos da neurociência cognitiva no capital de saberes de docentes que atuam na educação infantil e nos anos iniciais do ensino fundamental. Dissertação (Mestrado em Educação) - Universidade Regional Integrada do Alto Uruguai e das Missões, Frederico Westphalen, 2016.

SIQUEIRA, C. M.; GURGEL-GIANNETTI, J. Mau desempenho escolar: uma visão atual. Revista da Associação Médica Brasileira, São Paulo, v. 57, n. 1, p. 78-87, jan./fev. 2011. 
CORREAA, Thiago Henrique Barnabé; CELY, Claudia Patricia Moreno; CUADROS, Nubia Elena Pineda de. Reflexiones sobre el desarrollo del aprendizaje y la complejidad neurocognitiva.

Thiago Henrique Barnabé Corrêa

Universidade Federal do Triângulo Mineiro - UFTM | Instituto de

Ciências Exatas, Naturais e Educação

Uberaba | MG | Brasil. Contato: correa.uftm@gmail.com

ORCID 0000-0001-7123-1074

Claudia Patricia Moreno Cely

Universidad Pedagógica y Tecnologócia de Colombia - UPTC |

Facultad de Ciencias de la Educación

Tunja | Boyacá | Colômbia. Contato: claudia.moreno@uptc.edu.co

ORCID 0000-0002-7584-1897

Nubia Elena Pineda de Cuadros

Universidad Pedagógica y Tecnologócia de Colombia - UPTC |

Facultad de Estudios a Distancia

Tunja | Boyacá | Colômbia. Contato: nubia.pineda@uptc.edu.co

ORCID 0000-0001-8646-257X

Artigo recebido em: 30 dez. 2017 e aprovado em: 25 abr. 2018. 